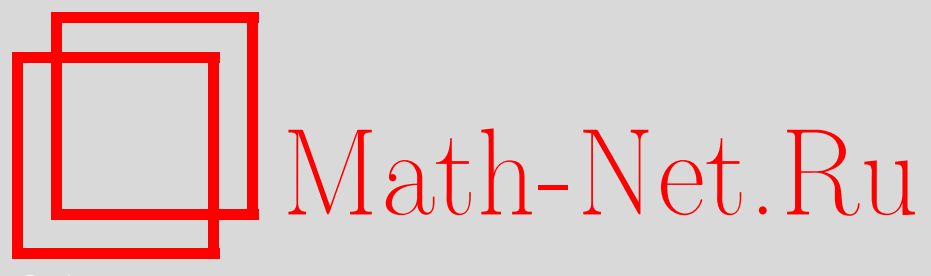

Д. А. Попов, Замечания о равномерных составных оценках осциллирующих интегралов с простыми особенностями, Изв. РАН. Сер. матем., 2008, том 72, выпуск 4, 173-196

DOI: https://doi.org/10.4213/im1141

Использование Общероссийского математического портала Math-Net.Ru подразумевает, что вы прочитали и согласны с пользовательским соглашением http://www . mathnet.ru/rus/agreement

Параметры загрузки:

IP: 3.82 .47 .9

26 апреля 2023 г., 18:22:14

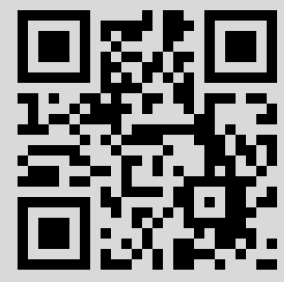




\section{Д. А. Попов \\ Замечания о равномерных составных оценках осциллирующих интегралов с простыми особенностями}

Рассматривается вопрос о построении асимптотически точных (при $\Omega \gg 1)$ и равномерных по параметрам $t=\left(t_{1}, t_{2}, \ldots, t_{m}\right)$ оценок осциллирующих интегралов, содержащих большой параметр $\Omega$. Предложен возможный многомерный аналог известной оценки И. М. Виноградова для одномерных интегралов. На основе этого предположения получены оценки интегралов с особенностями типа $A_{k}, D_{4}^{ \pm}$по классификации В. И. Арнольда и на примере особенностей $D_{5}^{ \pm}$обсуждается возможность обобщения полученных результатов.

Библиография: 28 наименований.

\section{§ 1. Введение}

Осциллирующим интегралом (ОИ) будем называть $n$-мерный интеграл вида

$$
I_{n}[u \mid t, \Omega]=\int_{M} e^{i \Omega \Phi(z, t)} u(z, t) d z .
$$

Здесь $z=\left(z_{1}, \ldots, z_{n}\right) \in \mathbb{R}^{n}, d z=d z_{1} \ldots d z_{n}$ и для определенности будем считать, что $M-n$-мерный параллелепипед, $M=\left\{z \in \mathbb{R}^{n}, a_{i} \leqslant z_{i} \leqslant b_{i}\right\}$. Принимающая действительные значения функция $\Phi$ называется фазой, функция $u$ - амплитудой и величина $\Omega$ - болъшим параметром. Ниже всегда рассматривается семейство фаз и амплитуд, зависящих от $m$-мерного параметра $t=\left(t_{1}, \ldots, t_{m}\right) \in \mathbb{R}^{m}$, хотя для сокращения иногда вместо $\Phi(z, t), u(z, t)$ используется запись $\Phi(z), u(z)$.

В теории ОИ основными являются две следующие задачи:

1) об асимптотике ОИ (1.1) при $\Omega \rightarrow \infty$;

2 ) о равномерной по параметру $t \in \mathbb{R}^{m}$ оценке сверху величины $\left|I_{n}[u \mid t, \Omega]\right|$ при больших $\Omega$.

Задача 1) возникла в физике при исследовании явления дифракции. В этой задаче рассматриваются асимптотики интеграла $I_{n}[u \mid t, \Omega]$ при $\Omega \rightarrow \infty$ и фиксированном значении параметра $t, t=t_{0} . \mathrm{K}$ асимптотической оценке ОИ сводятся многие вопросы теории распространения волн, квазиклассического приближения в квантовой механике и теории интегральных операторов Фурье. Метод стационарной фазы (СФ) в сочетании с методами теории особенностей в принципе решают задачу 1) в большинстве интересных случаев (см. [1]-[7]).

В настоящей работе мы рассмотрим задачу 2). Эта задача допускает различные формулировки. В частности, формулировка, принятая в теории особенностей, будет кратко обсуждаться ниже. Постановка задачи 2), рассматриваемая в настоящей работе, возникла в связи с приложениями равномерных оценок 
в теории чисел, спектральной теории и современном гармоническом анализе. Вопрос о равномерных оценках ОИ был впервые поставлен в теории чисел. В работах И. М. Виноградова и Дж. Ван дер Корпута (см. [8]-[10]) были даны первые нетривиальные оценки одномерных ОИ и предложен элементарный метод их вывода (по поводу дальнейшего развития этого метода и его применения см. работы [11]-[13]). В настоящее время равномерные оценки ОИ находят все более широкое применение в вышеперечисленных областях и ряде других направлений (см., например, работы [14]-[19]).

Рассматриваемая ниже постановка задачи о равномерных оценках ОИ была предложена в работе [18]. В этой постановке рассматривается разбиение интересующей нас области $T$ параметров $t \in T$ на зависящие от большого параметра $\Omega$ подобласти, в каждой из которых используется своя элементарная оценка. Используемый метод и его связь с методами работ [11]-[13] и методом стационарной фазы будут описаны ниже. Здесь отметим следующее. Как правило, в указанных выше приложениях оценка ОИ является промежуточным результатом и в дальнейшем полученную оценку необходимо интегрировать (суммировать) по области $T$. При этом область $T$, как правило, некомпактна и интерес представляют максимально точные оценки. Рассматриваемые разбиения области $T$ связаны с геометрией ее пересечения с каустикой $\mathcal{K}=\mathcal{K}[\Phi]$ фазы $\Phi$. Каустика разбита на подобласти с различными типами вырожденных критических точек. Ширины трубчатых окрестностей этих подобластей, где необходимо учитывать вырождение, зависят от величины $\Omega$. Это и приводит к рассматриваемым ниже разбиениям области параметров $T$.

Рассмотрим ОИ (1.1) с гладким семейством фаз $\Phi(z, t)$. О величине $\Omega \geqslant$ 1 не делается никаких априорных предположений, и все ограничения снизу величины $\Omega$ получаются в ходе вывода оценки. При этом, хотя явно это не указывается, фаза и амплитуда могут зависеть от $\Omega$. Задача состоит в оценке сверху величины $\left|I_{n}[u \mid t, \Omega]\right|$ при больших $\Omega$ и всех $t$ из некоторого множества $T \subset \mathbb{R}^{m}$. В соответствии с работой [18] ниже будет рассматриваться задача построения элементарных составных оценок интеграла (1.1). По определению это оценки вида

$$
\left|I_{n}[u \mid t, \Omega]\right| \leqslant C_{i n}[u, t] \Omega^{-\alpha_{i}+\varepsilon}, \quad \alpha_{i}>0,
$$

справедливые при всех $t \in T_{i} \subset T$ и любых $\varepsilon>0$ при условиях $\Omega \geqslant \Omega_{\text {in }}(t)$. При этом константы $C_{i n}[u, t]$ и величины $\Omega_{i n}(t)$ должны быть явно указаны и множества $T_{i}$ должны покрывать множество $T: T=\bigcup_{i} T_{i}$. Отметим, что возникающее при построении оценок разбиение $T=\bigcup_{i} T_{i}$ зависит от $\Omega$ и эта зависимость следует из условий $\Omega \geqslant \Omega_{\text {in }}(t)$.

Если элементарные составные оценки рассматривать с точностью до не зависящих от параметров $t$ и $\Omega$ мультипликативных констант $C_{i n}[u]$, то эти оценки можно упорядочить по их качеству. Пусть для любого $t \in T$ имеются две такие оценки. Тогда первая считывается лучше второй, если она сильнее второй при $\Omega \rightarrow \infty$ в области $T^{\prime} \subset T$ и не слабее второй для любого $t \in T \backslash T^{\prime}$. Это дает возможность определить понятие асимптотически точной (неулучшаемой) для любого $t \in T$ оценки. Для такой оценки верхнюю грань величины $\alpha_{i}$ в формуле (1.2) дает метод СФ. Задача состоит в построении оценок, максимально близких к асимптотически точным, т. е. оценок, для которых объем области 
в пространстве параметров, где оценка не является асимптотически точной, был как можно меньше и в любом случае меньше $\Omega^{-\gamma}, \gamma>0$.

Примеры таких равномерных составных оценок рассмотрены в работе [18]. Простейшая из них возникает при оценке интеграла Эйри. Это одномерный ОИ $I_{1}[u \mid t, \Omega]$, в котором $a_{1}=-\infty, b_{1}=+\infty, u \in C_{0}^{\infty}, \Phi(x, t)=\frac{1}{3} x^{3}-t x, z_{1}=x$ и $T=[0,1]$. В работе [18] для этого интеграла получена составная оценка вида

$$
\left|I_{1}[u \mid t, \Omega]\right| \leqslant C[u] \cdot \begin{cases}\Omega^{-1 / 2} t^{-1 / 4}, & \Omega^{-2 / 3} \leqslant t \leqslant 1 \\ \Omega^{-1 / 3}, & 0 \leqslant t \leqslant \Omega^{-2 / 3}\end{cases}
$$

соответствующая разбиению $T=T_{1} \cup T_{2}$, в котором $T_{1}=\left[0, \Omega^{-2 / 3}\right], T_{2}=$ $\left[\Omega^{-2 / 3}, 1\right]$. Эта оценка асимптотически точна, так как справа в формуле $(1.3)$ стоят первые члены асимптотики интеграла Эйри при $t>0$ и $t=0$, т. е. оценка определяется вкладом критических точек. Кроме того, эта составная оценка согласована, так как две оценки в правой части формулы (1.3) совпадают (с точностью до констант $C[u]$ ) при $t=\Omega^{-2 / 3}$.

Заметим, что для интеграла Пирси - аналога интеграла Эйри, в котором $\Phi(x, t)=\frac{x^{4}}{4}+\frac{1}{2} a x^{2}+b x, t=(a, b),-$ по-видимому уже нельзя получить асимптотически точную элементарную составную оценку в круге $a^{2}+b^{2} \leqslant 1$ (точную в каждой точке этого круга [18]).

В соответствии с методом СФ основную роль при оценке ОИ будут играть критические точки фазы $\Phi(z)$, которые ниже обозначаются через $z_{c} \equiv z_{c}(t)$. Матрица вторых производных фазы $\Phi$ в точке $z_{c}$ (гессиан) будет обозначаться через $\Phi_{c}^{(2)} \equiv \Phi^{(2)}\left(z_{c}, t\right)$. Если для любого $t \in T$

$$
\operatorname{corank} \Phi_{c}^{(2)} \equiv n-\operatorname{rank} \Phi_{c}^{(2)} \leqslant 1,
$$

то фаза $\Phi$ при $t \in T$ может иметь особенности только типа $A_{k}[3],[20]$, [21]. В этом случае для получения элементарных составных оценок, близких к асимптотически точным, для любого $t \in T$ можно воспользоваться результатами работы [18]. Если же при некоторых $t \in T$ имеются критические точки $z_{c}(t)$ такие, что corank $\Phi_{c}^{(2)} \geqslant 2$, то фаза имеет более сложные особенности.

Основные результаты об оценках ОИ с использованием теории особенностей получены в работах [3], [4], [22]-[26]. Принятая в этих работах постановка задачи о равномерных оценках ОИ состоит в следующем. Пусть при заданном $t=t_{0} \in T$ функция $\Phi_{0}(z)=\Phi\left(z, t_{0}\right)$ имеет особенность типа $\nu$. Предполагается, что носитель амплитуды $u$ лежит в достаточно малом шаре $\left|z-z_{c}\left(t_{0}\right)\right| \leqslant \sigma_{1}$. Теория особенностей в принципе позволяет по типу $\nu$ найти вклад критической точки $z_{c}\left(t_{0}\right)$ в асимптотику при $\Omega \rightarrow \infty$ интеграла (1.1). Пусть главный член этого вклада имеет вид

$$
I_{n}^{0}\left[u \mid z_{c}\left(t_{0}\right)\right]=\frac{C[u](\ln \Omega)^{\gamma_{\nu}}}{\Omega^{\beta_{\nu}} F_{\nu}\left(z_{c}\left(t_{0}\right)\right)} .
$$

Тогда возникает следующий вопрос: будет ли в шаре $\left|t-t_{0}\right| \leqslant \sigma_{2}$ при достаточно малом $\sigma_{2}$ верна оценка

$$
\left|I_{n}[u \mid t, \Omega]\right| \leqslant C_{n}[u, t] \Omega^{-\beta_{\nu}+\varepsilon} \quad \forall \varepsilon>0 .
$$


В указанных выше работах получен положительный ответ на этот вопрос в семействах фаз общего положения, зависящих от $m \leqslant 7$ параметров, а в двумерном случае $(n=2)$ - при любом числе параметров. Показано [26], что уже при $n=3$ имеется особенность высокой коразмерности, для которой ответ на поставленный вопрос отрицателен.

Рассмотрим вопрос: можно ли использовать эти результаты для получения нужных нам составных оценок, близких к асимптотически точным? Как это ясно из рассмотренного выше примера интеграла Эйри, для получения таких оценок надо иметь достаточно точную информацию о зависимости констант $C_{n}[u, t]$ в оценке (1.5) от параметра $t$. Вопрос о вычислении констант в оценках (1.5) в теории особенностей по-видимому остается открытым. С другой стороны, если пользоваться оценками вида

$$
\left|I_{n}[u \mid t, \Omega]\right| \leqslant C_{n}[u] \Omega^{-\beta_{\nu}+\varepsilon}, \quad\left|t-t_{0}\right|<\sigma,
$$

то возникающее разбиение области $T$ не зависит от $\Omega$ и получаемая составная оценка может быть далека от точной.

В работе [18] для получения нужных оценок в случае особенностей типа $A_{k}$ $\left(\nu=A_{k}\right)$ использовалось сочетание метода СФ и элементарного метода оценок одномерных ОИ. Последний метод берет свое начало в работах И. М. Виноградова и Дж. Ван дер Корпута и получил свое развитие в работах [11]-[13]. В частности, в этих работах получен следующий результат. Пусть в одномерном ОИ (1.1) фаза $\Phi$ имеет $p \geqslant 2$ непрерывных производных (при $z \in \operatorname{supp} u$ ) и условие

$$
\left|\Phi^{(p)}(z, t)\right| \geqslant \Phi_{p}>0, \quad z \in \operatorname{supp} u,
$$

выполняется равномерно по $z, t$. Тогда имеет место оценка

$$
\left|I_{1}[u \mid t, \Omega]\right| \leqslant C_{p}[u]\left(\Omega \Phi_{p}\right)^{-1 / p}
$$

и константа $C_{p}[u]$ в ней может быть явно указана. Именно этот результат вместе с полученными в работе [18] оценками остаточных членов в разложении ОИ позволяет найти нужные оценки для многомерных ОИ с фазой, имеющей особенности типа $A_{k}$.

Цель настоящей работы - рассмотреть возможность обобщения указанного подхода в случае, когда фаза имеет вырожденные критические точки с более сложным вырождением.

Для нахождения нужных оценок в областях параметров, где имеются только простые особенности [3], [20], [21], достаточно получить двумерный аналог оценок (1.7), так как для простых особенностей corank $\Phi_{c}^{(2)} \leqslant 2$. Заметим, что все особенности в семействах фаз общего положения при $m \leqslant 5$ простые.

При наличии двумерного аналога оценки (1.7) получение нужных составных оценок в областях параметров, где все особенности простые, уже не представляет принципиальных трудностей и производится следующим образом.

1. Пусть критические точки $z_{c}(t), t \in T$, известны и все они простые. С помощью разбиения единицы и количественного варианта формулы интегрирования по частям [18, лемма 3.1] задача локализуется, т. е. сводится к исследованию $n$-мерных ОИ по малым (диаметром $\Omega^{-\gamma}, \gamma>0$ ) окрестностям критических точек. 
2. Делая, если нужно, линейную замену координат $y=A z$ в каждом таком интеграле, получим, что фаза как функция переменных $y_{2}, \ldots, y_{n}$ для особенностей типа $A_{k}$ или как функция переменных $y_{3}, \ldots, y_{n}$ для других простых особенностей имеет только невырожденные критические точки. Интегрируем по этим переменным, используя теорему 2.1 или следствие 2.1 из работы [18].

3. Оставшийся одномерный ОИ по переменной $y_{1}$ или двумерный по переменным $y_{1}, y_{2}$ оцениваем с помощью (1.7) или соответствующего двумерного аналога этой оценки.

Для особенностей типа $A_{k}$ эта схема реализована в работе [18]. Ясно, что она естественно обобщается и на непростые особенности, но в этом случае нужны трехмерные обобщения оценок (1.7) при corank $\Phi_{c}^{(2)} \geqslant 3$.

Чтобы сформулировать нужную нам гипотезу о многомерном аналоге оценки (1.7), рассмотрим сначала случай особенностей типа $A_{k}$. Тогда на этапе 3 оценки мы имеем одномерный ОИ с фазой $\widetilde{\Phi}(y), y=y_{1} \in \mathbb{R}$. Если $\widetilde{\Phi}^{(k)}\left(y_{c}\right)=0$, $k \leqslant p-1,\left|\widetilde{\Phi}^{(p)}\left(y_{c}\right)\right| \geqslant \Phi_{p}>0$, то условие (1.6) означает, что в точке $z_{c}$ имеется особенность типа $A_{p-1}$, а оценка (1.7) имеет вид (с точностью до не зависящей от $\Omega$ константы) первого члена асимптотики соответствующего одномерного интеграла с фазой $\widetilde{\Phi}$. Учитывая явный вид (1.4) основного вклада критической точки $z_{c}$ типа $\nu$ в асимптотику ОИ, можно предположить, что естественным обобщением условий (1.6) являются условия вида

$$
\left|F_{\nu}(z)\right| \geqslant B_{\nu}>0
$$

в которых функция $F_{\nu}(z)$ определяется равенством (1.4), и предполагаемая оценка интеграла $I_{n}[u \mid t, \Omega]$ при условии (1.8) может иметь вид

$$
\left|I_{n}[u \mid t, \Omega]\right| \leqslant \frac{C_{n, \nu}[u, t]}{\Omega^{\beta_{\nu}-\varepsilon} B_{\nu}} \quad \forall \varepsilon>0 .
$$

Основные результаты настоящей работы относятся к оценкам двумерных ОИ. Это не является дополнительным ограничением, так как мы рассматриваем простые особенности. Сформулированное выше предположение при $n=2$ будет доказано для особенностей типа $A_{k}$ и в несколько ослабленной форме для особенностей типа $D_{4}^{ \pm}$. Уже для особенностей типа $D_{5}^{ \pm}$полученная ниже оценка хотя и нетривиальна, но далека от асимптотически точной.

\section{§ 2. Невырожденные критические точки}

В этом параграфе будет приведено следствие из результатов, полученных в работе [18], позволяющее в $n$-мерном случае достаточно просто оценить ОИ, когда все критические точки невырождены. В $\S 3,4$ результаты настоящего параграфа не используются, но они позволяют строить оценки $n$-мерных ОИ без ссылок на работу [18]. Попутно будут введены нужные определения и обозначения.

Константой ниже называется любая величина, не зависящая от переменных $z$ и $\Omega$. Все константы, значения которых не указываются, обозначаются через $C$, и так могут обозначаться различные константы. Всегда указывается, от каких величин эти константы зависят, и если эти величины не указаны, 
то $C$ - абсолютная константа. Все константы, фигурирующие в тексте, эффективны, и метод их вычисления содержится в доказательстве соответствующей оценки.

Используются стандартные обозначения: $\partial_{i}=\partial / \partial z_{i}, \partial^{\alpha}=\partial_{1}^{\alpha_{1}} \ldots \partial_{n}^{\alpha_{n}}$, $|\alpha|=\alpha_{1}+\cdots+\alpha_{n},|z|$ - евклидова норма $z \in \mathbb{R}^{n}$ и $|t|$ - евклидова норма $t$ в пространстве параметров $\mathbb{R}^{m}$. Через $\partial^{k}$ обозначается любая производная $\partial^{\alpha}$ порядка $k,|\alpha|=k$.

Рассмотрим множество $T_{0}$ в пространстве параметров $\mathbb{R}^{m}$ такое, что при $t \in T_{0}$ фаза $\Phi$ имеет только конечное число $\mathcal{N} \geqslant 1$ невырожденных критических точек $z_{c} \equiv z_{c}(t) \in \operatorname{supp} u$. Таким образом, будем предполагать, что при $t \in T_{0}$

$$
\left|\operatorname{det} \Phi^{(2)}\left(z_{c}\right)\right| \geqslant \Phi_{2 c} \geqslant \Phi_{2}>0 \quad \forall z_{c} \in \operatorname{supp} u .
$$

Здесь и ниже $\Phi^{(2)}(z)$ - гессиан (матрица вторых производных $\left.\partial_{i} \partial_{j} \Phi\right)$ в точке $z$. Заметим, что условие $\left|\operatorname{det} \Phi^{(2)}(z)\right| \geqslant \Phi_{2}>0$ является многомерным обобщением условия (1.6) при $p=2$.

Чтобы сформулировать результат, введем некоторые определения. Будем говорить, что $(u, \Phi) \in \Lambda(v, \lambda), \lambda=\left(\lambda_{0}, \lambda_{1}, \lambda_{2}\right)$, если выполнены следующие условия:

1) $u \in C_{0}^{\infty}, u(z)=0$ при $|z| \geqslant 1$ и

$$
\left|\partial^{\alpha} u(z)\right| \leqslant v \lambda_{2}^{|\alpha|}, \quad \lambda_{2} \geqslant 1
$$

2) $\Phi \in C^{\infty}$ при $|z| \leqslant 1$ и

$$
\left|\partial^{\alpha} \Phi(z)\right| \leqslant \lambda_{0} \lambda_{1}^{|\alpha|}, \quad|\alpha| \geqslant 2, \quad \lambda_{1} \geqslant 1 .
$$

Заметим, что приведенные ниже результаты справедливы и при $u \in C_{0}^{k}$, $\Phi \in C^{k}$ для достаточно больших $k$ (см. замечание 1 в конце настоящего параграфа).

Пусть $\mu_{1}^{c}, \ldots, \mu_{n}^{c}-$ собственные числа гессиана $\Phi^{(2)}\left(z_{c}\right)$ и

$$
B_{c}=\min _{k}\left|\mu_{k}^{c}\right|, \quad \min _{z_{c} \in \operatorname{supp} u} B_{c} \geqslant B_{0}>0 .
$$

Поскольку

$$
\begin{aligned}
\max _{k}\left|\mu_{k}^{c}\right| & \leqslant n \max _{i, j}\left|\partial_{i} \partial_{j} \Phi\left(z_{c}\right)\right| \leqslant n \lambda_{0} \lambda_{1}^{2}, \\
\left|\operatorname{det} \Phi^{(2)}\left(z_{c}\right)\right| & \leqslant\left(\max _{k}\left|\mu_{k}^{c}\right|\right)^{n-1} B_{c},
\end{aligned}
$$

то, следовательно (см. (2.1)),

$$
B_{c} \geqslant C_{n} \Phi_{2 c}\left(\lambda_{0} \lambda_{1}^{2}\right)^{-n+1} .
$$

Пусть $z_{\text {ех }} \neq z_{c}$ - экстремальные точки функции $G=|\nabla \Phi|^{2}, \nabla \equiv \nabla_{z}=$ $\left(\partial_{1} \ldots \partial_{n}\right)$, определяемые из уравнений $\partial_{i} G=0$. Определим константу $g>0$ из условия

$$
g \equiv g(t) \leqslant \min _{z_{\mathrm{ex}} \neq z_{c}}\left|G\left(z_{\mathrm{ex}}\right)\right|^{1 / 2}
$$


Заметим, что при $t \in T_{0}$ (см. [18])

$$
\left|z_{\mathrm{ex}}-z_{c}\right| \geqslant C_{n} B_{0}\left(\lambda_{0} \lambda_{1}^{3}\right)^{-1}, \quad\left|z_{c}-z_{c^{\prime}}\right| \geqslant C_{n} B_{0}\left(\lambda_{0} \lambda_{1}^{3}\right)^{-1},
$$

следовательно, для количества $\mathcal{N}$ критических точек в носителе амплитуды $u$, т. е. при $|z| \leqslant 1$, имеет место оценка

$$
\mathcal{N} \leqslant C_{n} \Phi_{2}^{-n}\left(\lambda_{0}^{n} \lambda_{1}^{2 n+1}\right)^{n}
$$

ТеОРема 2.1. Пусть выполнены следующие условия:

1) $(u, \Phi) \in \Lambda(v, \lambda)$;

2) при $t \in T_{0} \subset \mathbb{R}^{m}$ все критические точки $z_{c}$ невырождены $и$

$$
\left|\operatorname{det} \Phi^{(2)}\left(z_{c}\right)\right| \geqslant \Phi_{2}>0
$$

3) $\Omega \geqslant 1$ и выполняются оценки

$$
v \leqslant C \Omega^{\theta_{1}}, \quad 1 \leqslant \mathcal{N} \leqslant C \Omega^{\theta_{2}}, \quad \lambda_{0} \leqslant C \Omega^{\theta_{3}}, \quad \theta_{i} \geqslant 0 .
$$

Тогда можно указать константы $C_{n}(\lambda, \varepsilon), C_{n}(\varepsilon, \theta), \theta=\left(\theta_{1}, \theta_{2}, \theta_{3}\right)$, такие, что если при $t \in T_{0}$ для некоторого $\varepsilon, 0<\varepsilon \leqslant 1$, выполнено

$$
\Omega \geqslant C_{n}(\lambda, \varepsilon)\left[1+g^{-2-2 \varepsilon}+\Phi_{2}^{-6-6 \varepsilon}\right],
$$

где константа $g=g(t)$ определена в (2.6), то при $t \in T_{0}$ имеет место оченка

$$
\left|I_{n}[u \mid t, \Omega]\right| \leqslant \frac{C_{n}(\varepsilon, \theta) v}{\Omega^{n / 2}} \sum_{z_{c} \in \operatorname{supp} u}\left|\operatorname{det} \Phi^{(2)}\left(z_{c}\right)\right|^{-1 / 2} .
$$

ДокАЗАтельство. Покажем, что нужный результат вытекает из [18, следствие 2.1]. Введем обозначение

$$
\lambda_{3}=\max \left(\lambda_{1}, \lambda_{2}\right)
$$

и предположим, что

$$
\begin{gathered}
\lambda_{0} \Omega \geqslant C, \quad \lambda_{0}^{2} \lambda_{1}^{12} B_{0}^{-6} \leqslant C \Omega^{1-\varepsilon}, \\
\lambda_{0}^{2} \lambda_{3}^{6} B_{0}^{-3} \leqslant C \Omega^{1-\varepsilon}, \quad g \geqslant C_{n} \Omega^{-(1-\varepsilon) / 2} \lambda_{0}^{1 / 2} \lambda_{1},
\end{gathered}
$$

где $B_{0}$ - величина, определенная в формуле (2.4). При этих условиях в силу $\left[18\right.$, следствие 2.1] для любых $Q_{1} \geqslant 0, Q_{2} \geqslant 0$ имеет место оценка

$$
\begin{aligned}
& \left|I_{n}[u \mid t, \Omega]\right| \leqslant \sum_{z_{c} \in \operatorname{supp} u} \frac{v}{\Omega^{n / 2}\left|\operatorname{det} \Phi^{(2)}\left(z_{c}\right)\right|^{1 / 2}} \sum_{q=0}^{Q_{1}} C_{n q}\left(\frac{\lambda_{0}^{2} \lambda_{3}^{6}}{\Omega B_{c}^{3}}\right)^{q} \\
& \quad+C_{n}\left(Q_{1}, Q_{2}, \varepsilon, \theta\right)\left[\Omega^{-Q_{2}}+\sum_{z_{c} \in \operatorname{supp} u} \frac{v}{\Omega^{n / 2}\left|\operatorname{det} \Phi^{(2)}\left(z_{c}\right)\right|^{1 / 2}}\left(\frac{\lambda_{0}^{2} \lambda_{3}^{6}}{\Omega B_{c}^{3}}\right)^{Q_{1}+1}\right] .
\end{aligned}
$$

В соответствии с неравенством (2.5) и условиями теоремы константы $C_{n}(\lambda, \varepsilon)$ в (2.9) можно выбрать таким образом, чтобы выполнялись условия (2.12) и, 
следовательно, была справедлива оценка (2.13). Выбирая $Q_{1}=0$ и учитывая неравенства (2.12), из (2.13) получаем

$$
\left|I_{n}[u \mid t, \Omega]\right| \leqslant C_{n}^{1}\left(Q_{2}, \varepsilon, \theta\right) \sum_{z_{c} \in \operatorname{supp} u} \frac{v}{\Omega^{n / 2}\left|\operatorname{det} \Phi^{(2)}\left(z_{c}\right)\right|}+\frac{C_{n}^{2}\left(Q_{2}, \varepsilon, \theta\right)}{\Omega^{Q_{2}}} .
$$

В силу условий $(2.8),(2.9)$ можно считать, что $\Phi_{2} \geqslant \Omega^{-1}$, и целое число $Q_{2}=$ $Q_{2}(\varepsilon, \theta)$ можно выбрать таким образом, что $\mathcal{N} v \Omega^{-n / 2} \Phi_{2}^{-1} \geqslant \Omega^{-Q_{2}}$. Нужный результат (2.10) теперь следует из оценки (2.14).

Из результатов работы [18] вытекает, что утверждение теоремы 2.1 остается верным, если $u \in C_{0}^{k}, \Phi \in C^{k}$ и условия (2.2), (2.3) выполняются при $|\alpha| \leqslant k$. При $\theta_{i}=\theta_{0}$ можно взять

$$
k \geqslant 2\left[\left(2 \theta_{0}+3\right)\left(Q_{2}+2 \theta_{0}\right) \varepsilon^{-2}\right]+n+3 .
$$

ЗАмЕчАниЕ 1. В формулировке теоремы 2.1 условия (2.9) можно заменить на условия (2.12). Условие, налагаемое на величину $g(2.6)$ и содержащееся в $(2.9)$, означает, что величина $\delta_{0}=C_{n} \Omega^{-(1-\varepsilon) / 2} \lambda_{0}^{1 / 2} \lambda_{1} B_{0}^{-1}$ достаточно мала [18]. Величина $\delta_{0} \leqslant C_{n} B_{0} \lambda_{0} \lambda_{1}^{3}$ называется достаточно малой, если минимум величины $G$ в области $\left|z-z_{c}\right| \geqslant \delta_{0}$ достигается на одной из сфер $\left|z-z_{c}\right|=\delta_{0}$ и условие для величины $g$ можно заменить требованием достаточной малости величины $\delta_{0}=C_{n} \Omega^{-(1-\varepsilon) / 2} \lambda_{0}^{1 / 2} \lambda_{1} B_{0}^{-1}$, задающей размер окрестностей критических точек $z_{c}$, дающих основной вклад в оценку величины $\left|I_{n}[u \mid t, \Omega]\right|$.

При доказательстве теоремы 2.1 нигде не предполагалось, что фаза $\Phi$ и амплитуда $u$ не зависят от $\Omega$. Такая зависимость допустима, важно только то, чтобы при этом выполнялись условия (2.12).

Граница области $T_{0}$ в пространстве параметров, где имеет место оценка (2.10), задается условием (2.9) (или (2.12)). Из этого условия следует ограничение снизу расстояния от точек $t \in T_{0}$ до каустики $\mathcal{K}$. Напомним, что каустика $\mathcal{K}$ это множество таких $t \in \mathbb{R}^{m}$, при которых фаза $\Phi$ имеет хотя бы одну вырожденную критическую точку $z_{c}^{0}$, в которой $\operatorname{det} \Phi^{(2)}\left(z_{c}^{0}\right)=0$.

Оценка (2.10) без условий (2.9) приведена в работе [22]. Условия (2.9) достаточны для справедливости этой оценки. Заметим, что условия (2.9) не являются точными, однако без условий такого типа оценка (2.10) не верна уже для интеграла Пирси [18].

\section{§ 3. Особенности типа $A_{k}$}

Начиная с этого параграфа и везде далее (кроме п. 3.2) будем предполагать, что $n=2$, т. е. рассматривать двумерные ОИ. В этом случае будут использоваться обозначения $z=(x, y)$ и

$$
I_{2}[u \mid t, \Omega] \equiv I_{2}[u \mid t] \equiv I_{2}[u]=\iint_{M} e^{i \Omega \Phi} u d x d y .
$$

Как уже указывалось, так как мы ограничиваемся рассмотрением простых особенностей, то $\operatorname{rank} \Phi^{(2)}\left(z_{c}\right) \geqslant n-2$ и предположение $n=2$ в сущности не является ограничением, так как по $n-2$ переменным можно интегрировать, 
используя теорему 2.1 или результаты работы [18]. Метод интегрирования по этим переменным описан в 1 и демонстрируется далее при доказательстве теоремы 3.1.

3.1. Формулировка результата. Вблизи каустики $\mathcal{K}$ оценка $(2.10)$ не обоснована, так как $\Phi_{2} \rightarrow 0$ при $d(t, \mathcal{K}) \rightarrow 0, d(t, \mathcal{K})$ - евклидово расстояние от точки $t$ до каустики, следовательно, нарушается условие (2.9). Чтобы рассмотреть простейший случай области $T_{1} \subset \mathbb{R}^{m}, T_{1} \cap \mathcal{K} \neq \varnothing$, будем предполагать, что

$$
\left|\partial_{x}^{2} \Phi(x, y)\right| \geqslant \Phi_{21}>0, \quad t \in T_{1}, \quad z \in \operatorname{supp} u .
$$

Тогда $\operatorname{rank} \Phi^{(2)}\left(z_{c}\right) \geqslant 1$ при $n=2$ и, исключая случай бесконечнократного вырождения, при $d(t, \mathcal{K}) \rightarrow 0, t \in T_{1}$, возникают только особенности типа $A_{k}$, $k \geqslant 2$. Условие $(3.2)$ так же, как и $\left|\operatorname{det} \Phi^{(2)}(z)\right|>0$, можно рассматривать как одно из двумерных обобщений условий (1.6) при $p=2$.

Предположим, что существует конечный набор гладких решений $x_{k}(y)$ уравнения

$$
\partial_{x} \Phi(x, y)=0, \quad k=1,2, \ldots, \mathcal{N} .
$$

В силу определения $x_{k}(y)$ - критические точки фазы $\Phi$, рассматриваемой как функция от $x$. Введем функции

$$
S_{k}(y)=\Phi\left(x_{k}(y), y\right)
$$

и предположим, что для некоторого $p_{k}, 2 \leqslant p_{k} \leqslant \bar{p}$,

$$
\left|S_{k}^{\left(p_{k}\right)}(y)\right| \geqslant S_{k}>0 .
$$

При условиях (3.2), (3.5) все критические точки $z_{c}$ имеют тип $A_{n}$ и $n \leqslant \bar{p}-1$.

Для заданной критической точки $x_{k}(y)$ и целого числа $q \geqslant 0$ определим функцию

$$
f_{q}^{k}(y)=D_{q}^{k}(y)\left|\partial_{x}^{2} \Phi\left(x_{k}(y), y\right)\right|^{-1 / 2},
$$

где

$$
\begin{gathered}
D_{q}^{k}(y)=\sum_{\mu=0}^{2 q} b_{\mu, \mu+q}^{k}, \\
b_{\mu, p}^{k}=\frac{i^{p+\mu}}{\mu ! p ! 2^{p}\left(\partial_{x}^{2} \Phi\left(x_{k}(y), y\right)\right)^{p}}\left[\left(\frac{\partial}{\partial x}\right)^{p}\left(u \Psi_{\mu}^{k}\right)\right]\left(x_{k}(y), y\right)
\end{gathered}
$$

и функции $\Psi_{\mu}^{k}$ задаются равенствами

$$
\Psi_{\mu}^{k}(x, y)=u(x, y)\left[\Phi(x, y)-\Phi\left(x_{k}(y), y\right)-\frac{1}{2} \partial_{x}^{2} \Phi\left(x_{k}(y), y\right)\left(x-x_{k}(y)\right)^{2}\right] .
$$

Величины $D_{q}^{k}$ входят в стандартный вид разложения ОИ

$$
I_{1}[u \mid y, t, \Omega]=\int e^{i \Omega \Phi(x, y)} u(x, y) d x
$$

по методу стационарной фазы [2], [18]. Переменная $y$ при этом рассматривается как параметр. То, что все критические точки $x_{k}(y)$ фазы $\Phi$ невырождены, следует из условий (3.2). Введем для интеграла (3.8) аналог $g_{1}$ величины $g(2.6)$. 
Если $x_{\mathrm{ex}} \equiv x_{\mathrm{ex}}(y)$ - экстремальные точки производной, определяемые из условий $\partial_{x}^{2} \Phi\left(x_{\mathrm{ex}}, y\right)=0, x_{\mathrm{ex}} \neq x_{k}(y)$, то

$$
g_{1} \equiv g_{1}(t)=\min _{x_{\mathrm{ex}} \neq x_{k}(y),|y| \leqslant 1}\left|\partial_{x} \Phi\left(x_{\mathrm{ex}}\right)\right| .
$$

Через $m[f]$ обозначим число участков монотонности функции $f$.

Tеорема 3.1. Пусть $(u, \Phi) \in \Lambda(v, \lambda)$ nри $t \in T_{1}$ и существует конечный набор гладких решений $x_{k}(y)$ уравнения (3.3). Пусть, кроме того, для некоторого $\varepsilon, 0<\varepsilon \leqslant 1$, выполняются условия

$$
\begin{gathered}
\Omega \geqslant C(\lambda, \varepsilon)\left(1+g_{1}^{-2-2 \varepsilon}+\Phi_{21}^{-3-3 \varepsilon}\right), \\
m\left[f_{q}^{k}\right] \leqslant C_{q} \Omega^{\varepsilon q},
\end{gathered}
$$

где функиии $f_{q}^{k}$ определень в (3.6), величина $\Phi_{21}$ - в (3.2) и $m\left[f_{q}^{k}\right]-$ число участков монотонности функиии $f_{q}^{k}$. Тогда при $t \in T_{1}$ имеет место равномерная по t оценка двумерного ОИ (3.1):

$$
\left|I_{2}[u \mid t]\right| \leqslant \sum_{x_{k}(y)} \frac{C_{k}(\varepsilon, \lambda) v}{\left(\Omega \Phi_{21}\right)^{1 / 2}\left(\Omega S_{k}\right)^{1 / p_{k}}} .
$$

Здесь суммирование ведется по всем решениям $x_{k}(y)$ уравнения (3.3).

Для доказательства теоремы 3.1 нам будут нужны вспомогательные леммы, доказательство которых приведено в п. 3.2. Эти леммы являются некоторым усилением соответствующих результатов, полученных в работе [18]. Это усиление позволяет вместо степени $-6-6 \varepsilon$ для $\Phi_{2}$ в $(2.9)$ получить степень $-3-3 \varepsilon$ для $\Phi_{21}$ в $(3.10)$, что существенно используется в $\S 4$.

3.2. Вспомогательные леммы. Первая из приведенных ниже лемм уточняет лемму 3.1 из работы [18] в случае, когда все вторые производные $\partial^{2} \Phi$ малы, и дает оценку степеней $\left(A_{\Phi}\right)^{N}$ оператора $A_{\Phi}$, определяемого равенствами

$$
\begin{aligned}
& A_{\Phi} f=\sum_{i=1}^{n} \partial_{i}\left(f G^{-1} \partial_{i} \Phi\right), \\
& G=|\nabla \Phi|^{2}=\sum_{i=1}^{n}\left(\partial_{i} \Phi\right)^{2}
\end{aligned}
$$

и входящего в формулу интегрирования по частям

$$
I_{n}[f \mid t, \Omega]=\left(\frac{i}{\Omega}\right)^{N} I_{n}\left[\left(A_{\Phi}\right)^{N} f \mid t, \Omega\right],
$$

справедливую для достаточно гладких $f$ при $z_{c} \notin \operatorname{supp} f, \operatorname{supp} f \subset M \backslash \partial M$.

В формулировке следующей леммы используются обозначения

$$
\begin{gathered}
\sum_{(k, l) \in O_{1}} a_{k l} \equiv \sum_{0 \leqslant k \leqslant N / 2} \sum_{l=0}^{k} a_{k l}+\sum_{k \geqslant N / 2}^{N} \sum_{l=0}^{N-k} a_{k l}, \\
\sum_{(k, l) \in O_{2}} a_{k l} \equiv \sum_{k \geqslant N / 2}^{N} \sum_{l=N-k}^{k} a_{k l},
\end{gathered}
$$


и через $\left|\partial^{k} f\right|,\left|\partial^{k} \Phi\right|, \Phi_{1}$ обозначены любые функции или константы такие, что выполняются оценки

$$
\begin{gathered}
\left|\partial^{\alpha} f\right| \leqslant\left|\partial^{k} f\right|, \quad\left|\partial^{\alpha} \Phi\right| \leqslant\left|\partial^{k} \Phi\right|, \quad|\alpha|=k \\
|\nabla \Phi|=G^{1 / 2} \geqslant \Phi_{1} .
\end{gathered}
$$

ЛЕмма 3.1. Пусть функиии $f, \Phi$ нужное число раз дифферениируемы, имеют место оченки (3.15) u $\left|\partial^{k} \Phi\right| \leqslant \lambda_{0} \lambda_{1}^{k}, k \geqslant 2$. Тогда для любого $N \geqslant 2$

$$
\begin{gathered}
\left|\left(A_{\Phi}\right)^{N} f\right| \leqslant C_{N, n}\left(\Phi_{1}\right)^{-2 N}\left[\sum_{(k, l) \in O_{1}}\left(\Phi_{1}\right)^{k}\left|\partial^{k-l} f\right|\left|\partial^{2} \Phi\right|^{N-k-l} \lambda_{0}^{l} \lambda_{1}^{3 l}\right. \\
\left.+\sum_{(k, l) \in O_{2}}\left(\Phi_{1}\right)^{k}\left|\partial^{k-l} f\right| \lambda_{0}^{N-k} \lambda_{1}^{2 N-2 k+l}\right] .
\end{gathered}
$$

ДокАЗАТЕЛЬСтво. Из [18, лемма 3.1] следует, что

$$
\left|\left(A_{\Phi}\right)^{N} f\right| \leqslant C_{N, n} \sum_{p=0}^{N}\left|\partial^{p} f\right| \sum_{j=0}^{N-p}\left(\Phi_{1}\right)^{-N-j} \sum_{(n)}\left|\partial^{2} \Phi\right|^{n_{2}} \ldots\left|\partial^{N+1} \Phi\right|^{n_{N+1}},
$$

и в этой формуле суммирование ведется по всем наборам $(n)=\left(n_{2}, \ldots, n_{N+1}\right)$ целых чисел таких, что $n_{i} \geqslant 0$,

$$
n_{2}+n_{3}+\cdots+n_{N+1}=j, \quad n_{2}+2 n_{3}+\cdots+N_{n_{N+1}}=N-p .
$$

Делая замену переменных $(j, p) \rightarrow(k, l), j=N-k, p=k-l$, перепишем неравенство (3.17) в виде

$$
\left|\left(A_{\Phi}\right)^{N} f\right| \leqslant C_{N, n} \sum_{k=0}^{N}\left(\Phi_{1}\right)^{-2 N+k} \sum_{l=0}^{k}\left|\partial^{k-l} f\right| \sum_{(n)}\left|\partial^{2} \Phi\right|^{n_{2}} \ldots\left|\partial^{N+1} \Phi\right|^{n_{N+1}},
$$

где набор $(n)$ удовлетворяет условиям

$$
n_{2}+\cdots+n_{N+1}=N-k, \quad n_{2}+2 n_{3}+\cdots+N n_{N+1}=N-k+l .
$$

Таким образом, при $N \geqslant 2$ имеют место неравенства $n_{3}+n_{4}+\cdots+n_{N+1} \leqslant l$ и $t_{0} \leqslant n_{2} \leqslant N-k$, где $t_{0}=\max (0, N-k-l)$. Выделяя суммирование по $n_{2}=t$, имеем

$$
\left|\left(A_{\Phi}\right)^{N} f\right| \leqslant C_{N, n} \sum_{k=0}^{N}\left(\Phi_{1}\right)^{-2 N+k} \sum_{l=0}^{k}\left|\partial^{k-l} f\right| \sum_{t=t_{0}}^{N-k}\left|\partial^{2} \Phi\right|^{t} \sum_{(n)}^{1}\left|\partial^{3} \Phi\right|^{n_{3}} \ldots\left|\partial^{N+1} \Phi\right|^{n_{N+1}},
$$

суммирование в $\sum_{(n)}^{1}$ ведется по наборам $(n)$ таким, что

$$
n_{3}+\cdots+n_{N+1}=N-k+t, \quad 2 n_{3}+\cdots+N n_{N+1}=N-k+l-t .
$$

Используя оценку $\left|\partial^{k} \Phi\right| \leqslant \lambda_{0} \lambda_{1}^{k}, k \geqslant 3$, получим

$$
\left|\left(A_{\Phi}\right)^{N} f\right| \leqslant C_{N, n} \sum_{k=0}^{N}\left(\Phi_{1}\right)^{-2 N+k} \sum_{l=0}^{k}\left|\partial^{k-l} f\right| \sum_{t=t_{0}}^{N-k}\left|\partial^{2} \Phi\right|^{t} \lambda_{0}^{N-k-t} \lambda_{1}^{2 N-2 k-2 t+l} .
$$


Поскольку $\left|\partial^{2} \Phi\right| \leqslant \lambda_{0} \lambda_{1}^{2}$, то

$$
\left|\left(A_{\Phi}\right)^{N} f\right| \leqslant C_{N, n} \sum_{k=0}^{N}\left(\Phi_{1}\right)^{-2 N+k} \sum_{l=0}^{k}\left|\partial^{k-l} f\right|\left|\partial^{2} \Phi\right|^{t_{0}} \lambda_{0}^{N-k-t_{0}} \lambda_{1}^{N-k-2 t_{0}+l} .
$$

Остается заметить, что $t_{0}=0$ при $(k, l) \in O_{2}$ и $t_{0}=N-k-l$ при $(k, l) \in O_{1}$, чтобы из (3.18) получить нужный результат (3.16).

Лемма 3.1 позволяет в одномерном случае уточнить следствие 2.1 из работы [18]. Это уточнение содержится в следующей лемме.

Лемма 3.2. Рассмотрим одномерный ОИ вида (3.8). Пусть $(u, \Phi) \in \Lambda(v, \lambda)$, выполняются условия (2.8), все критические точки базы $\Phi(x)$ невырождены и

$$
\left|\Phi^{(2)}\left(x_{c}\right)\right| \geqslant \Phi_{2 c} \geqslant \Phi_{2}>0 .
$$

Пусть, кроме того, для некоторого $\varepsilon, 0<\varepsilon \leqslant 1$, выполняются условия

$$
\begin{gathered}
\lambda_{0}^{2} \lambda_{3}^{6} \Phi_{2}^{-3} \leqslant C \Omega^{1-\varepsilon}, \quad \lambda_{2} \leqslant C \Omega^{(1-\varepsilon) / 2} \Phi_{2}^{1 / 2}, \quad \lambda_{0} \Omega \geqslant 1, \\
g \geqslant C \Omega^{-(1-\varepsilon) / 2} \Phi_{2}^{1 / 2},
\end{gathered}
$$

в которых величины $g, \lambda_{3}$ определены равенствами (2.6), (2.11). Тогда для любого $Q \geqslant 0$ выполняется равенство

$$
I_{1}[u \mid t, \Omega]=\sum_{x_{c}} I_{1 c}^{Q}+\Delta I_{1 Q}
$$

¿əe

$$
\begin{gathered}
I_{1 c}^{Q}=\left(\frac{2 \pi}{\Omega}\right)^{1 / 2} \frac{\exp \left\{\frac{i \pi}{4} \varkappa_{c}+i \Omega \Phi\left(x_{c}\right)\right\}}{\left|\Phi^{(2)}\left(x_{c}\right)\right|^{1 / 2}} \sum_{q=0}^{Q} \frac{D_{q}^{c}}{\Omega^{q}}, \\
\varkappa_{c}=\operatorname{sign} \Phi^{(2)}\left(x_{c}\right),
\end{gathered}
$$

и величины $D_{q}^{c}$ задаются равенствами (3.7), в которых индекс $k$ надо заменить на с и $x_{k}(y)$ - на $x_{c}$. При этом для величин $D_{q}^{c}, \Delta I_{1 Q}$ имеют место следующие ощенки:

$$
\begin{gathered}
\left|D_{q}^{c}\right| \leqslant C_{q} v\left(\frac{\lambda_{0}^{2} \lambda_{3}^{6}}{\Phi_{2 c}}\right)^{q}, \\
\left|\Delta I_{1 Q}\right| \leqslant \frac{C_{Q}(\varepsilon, \theta) v}{\left(\Omega \Phi_{2}\right)^{1 / 2}} \mathcal{N} \Omega^{-\varepsilon(Q+1)} .
\end{gathered}
$$

ДокАзАтельство. Лемма доказывается точно так же, как следствие 2.1 из работы [18], только для оценки величин $\left(A_{\Phi}\right)^{N} f$ теперь используется лемма 3.1.

3.3. Доказательство теоремы 3.1. Запишем интеграл $I_{2}[u](3.1)$ в виде повторного интеграла:

$$
I_{2}[u]=\int I_{1}[u] d y, \quad I_{1}[u]=\int u e^{i \Omega \Phi} d x .
$$


Условия (3.10) влекут выполнение условий (3.19) (см. §1), и по лемме 3.2 имеем

$$
I_{1}[u]=\sum_{x_{k}(y)}\left(\frac{2 \pi}{\Omega}\right)^{1 / 2} \frac{\exp \left\{\frac{i \pi}{4} \varkappa_{k}+i \Omega S_{k}(y)\right\}}{\left|\partial_{x}^{2} \Phi\left(x_{k}(y), y\right)\right|^{1 / 2}} \sum_{q=0}^{Q} \frac{D_{q}^{k}}{\Omega^{q}}+\Delta I_{1 Q} .
$$

Подставляя это выражение в равенство (3.23), получим

$$
I_{2}[u]=\sum_{x_{k}(y)}\left(\frac{2 \pi}{\Omega}\right)^{1 / 2} \exp \left\{\frac{i \pi}{4} \varkappa_{k}\right\} \sum_{q=0}^{Q} \int_{-1}^{1} f_{q}^{k}(y) e^{i \Omega S_{k}(y)} d y+\Delta I_{2 Q}
$$

и с помощью оценки (3.22) находим оценку остаточного члена

$$
\left|\Delta I_{2 Q}\right| \leqslant \int_{-1}^{1}\left|\Delta I_{1 Q}\right| d y \leqslant \frac{C_{Q}(\varepsilon, \theta)}{\left(\Omega \Phi_{2}\right)^{1 / 2}} v \mathcal{N} \Omega^{-\varepsilon(Q+1)} .
$$

Для оценки одномерных интегралов по у в правой части равенства (3.24) используем оценку (1.7) при $p=p_{k}$ (целые числа $p_{k}$ были определены выше, см. (3.5)). Согласно работе [14] в оценке (1.7) константу $C_{p}[u]$ можно записать в виде $C_{p}[u]=C m[u] \max |u|$, где $m[u]$ - число участков монотонности функции $u$. Из оценки (3.21) следует, что

$$
\left|f_{q}^{k}(y)\right| \leqslant \frac{C_{k q} v}{\left(\Phi_{21}\right)^{1 / 2}}\left(\frac{\lambda_{0}^{2} \lambda_{3}^{6}}{\Phi_{21}^{3}}\right)^{q}
$$

и, таким образом,

$$
\left|\int_{-1}^{1} f_{q}^{k}(y) e^{i \Omega S_{k}(y)} d y\right| \leqslant C_{k q} \frac{m\left[f_{q}^{k}\right] v}{\left(\Phi_{21}\right)^{1 / 2}\left(\Omega S_{k}\right)^{1 / p_{k}}}\left(\frac{\lambda_{0}^{2} \lambda_{3}^{6}}{\Phi_{21}^{3}}\right)^{q} .
$$

Используя эту оценку в равенстве (3.24) и учитывая оценку (3.25), получим

$$
\begin{gathered}
\left|I_{2}[u]\right| \leqslant \frac{1}{\left(\Omega \Phi_{2}\right)^{1 / 2}} \sum_{x_{k}(y)} \sum_{q=0}^{Q} \frac{C_{k q} m\left[f_{q}^{k}\right] v}{\left(\Omega S_{k}\right)^{1 / p_{k}}}\left(\frac{\lambda_{0}^{2} \lambda_{3}^{6}}{\Phi_{21}^{3}}\right)^{q} \\
+C_{Q}(\varepsilon, \theta) v \mathcal{N}\left(\Omega \Phi_{2}\right)^{-1 / 2} \Omega^{-\varepsilon(Q+1)} .
\end{gathered}
$$

Для завершения доказательства теперь достаточно воспользоваться условиями (3.10), (3.1) и выбрать нужным образом величину $Q=Q(\varepsilon, \theta)$.

Теорема 3.1 содержит трудно проверяемое условие (3.11). Заметим, однако, что в силу принципа локализации эта теорема реально применяется в случаях, когда носитель амплитуды $u$ мал, меньше $\Omega^{-\gamma}, \gamma>0$. С другой стороны, от условия (3.11) можно вообще избавиться ценой некоторого ухудшения оценки в области, где $\Phi_{21}<\Omega^{-\gamma}, \gamma>0$. Покажем, как это сделать. Рассмотрим одномерный ОИ

$$
I_{1}[f]=\int_{-1}^{1} f(y) e^{i \Omega S(y)} d y, \quad f \in C_{0}^{\infty}, \quad \operatorname{supp} f \in(-1,1),
$$

и запишем его в виде

$$
I_{1}[f]=\int_{-1}^{1} f^{(1)}(y)\left[\int_{y}^{1} e^{i \Omega S(t)} d t\right] d y .
$$


Таким образом, имеется оценка

$$
\left|I_{1}[f]\right| \leqslant \int_{-1}^{1}\left|f^{(1)}(y)\right| d y \cdot \max _{|y| \leqslant 1}\left|\int_{y}^{1} e^{i \Omega S(y)} d y\right| .
$$

Предполагая, что $\left|S^{(p)}(y)\right| \geqslant S_{p}, p \geqslant 2$, и используя оценку (1.7) вместе с указанным выше видом константы в ней, получим

$$
\left|I_{1}[f]\right| \leqslant \max _{|y| \leqslant 1}\left|f^{(1)}(y)\right| \frac{C_{p}}{\left(\Omega S_{p}\right)^{1 / p}} .
$$

Нас интересует случай $f=f_{q}^{k}(y)$. Чтобы оценить производные $\left(f_{q}^{k}\right)^{(1)}$, сначала оценим величины $d x_{k} / d y$. Дифференцируя тождества $\partial_{x} \Phi\left(x_{k}(y), y\right)=0$ по $y$, заключаем, что

$$
\frac{d x_{k}}{d y}=-\frac{\partial_{x} \partial_{y} \Phi\left(x_{k}(y), y\right)}{\partial_{x}^{2} \Phi\left(x_{k}(y), y\right)} .
$$

Отсюда следует оценка

$$
\left|\frac{d x_{k}}{d y}\right| \leqslant \lambda_{0} \lambda_{1}^{2} \Phi_{21}^{-1}
$$

используя которую, получим

$$
\left|\left(f_{q}^{k}\right)^{(1)}(y)\right| \leqslant \frac{C_{k_{q}} v}{\left(\Phi_{21}\right)^{1 / 2}}\left(\frac{\lambda_{0}^{2} \lambda_{3}^{6}}{\left(\Phi_{21}\right)^{3}}\right)^{q} \frac{\lambda_{2} \lambda_{1}^{4} \lambda_{3}}{\left(\Phi_{21}\right)^{2}} .
$$

С помощью этой оценки и (3.28) имеем вместо (3.26) оценку

$$
\left|\int f_{q}^{k}(y) e^{i \Omega S_{k}(y)} d y\right| \leqslant \frac{C_{k q} v}{\left(\Phi_{21}\right)^{1 / 2}\left(\Omega S_{k}\right)^{1 / p_{k}}}\left(\frac{\lambda_{0}^{2} \lambda_{3}^{6}}{\left(\Phi_{21}\right)^{3}}\right)^{q} \frac{\lambda_{2} \lambda_{1}^{4} \lambda_{3}}{\left(\Phi_{21}\right)^{2}},
$$

следовательно, вместо (3.12) - оценку

$$
\left|I_{2}[u]\right| \leqslant \sum_{x_{k}(y)} \frac{C_{k}(\varepsilon, \theta) v}{\left(\Omega \Phi_{21}\right)^{1 / 2}\left(\Omega S_{k}\right)^{1 / p_{k}}} \frac{\lambda_{2} \lambda_{1}^{4} \lambda_{3}}{\left(\Phi_{21}\right)^{2}} .
$$

Оценка (3.30) верна уже без условия (3.11).

Заметим, что равенства (3.29) позволяют выписать явный вид условий (3.5) в терминах ограничений на производные фазы $\Phi$. В качестве примера рассмотрим случай, когда $z_{c}=\left(x_{k}\left(y_{c}\right), y_{c}\right)$ является критической точкой типа $A_{2}$, т. е. для указанного номера $k$ выполнено $S_{k}^{(1)}\left(y_{c}\right)=S_{k}^{(2)}\left(y_{c}\right)=0, S_{k}^{(3)}\left(y_{c}\right) \neq 0$. Используя равенства (3.29), получим, что при условии $\partial_{x}^{2} \Phi\left(z_{c}\right) \neq 0$ выполнено

$$
\begin{aligned}
S_{k}^{(3)}\left(y_{c}\right)= & \left(\frac{\partial^{2} \Phi\left(z_{c}\right)}{\partial x^{2}}\right)^{-2}\left[\frac{\partial^{3} \Phi}{\partial x^{3}} \frac{\partial^{2} \Phi}{\partial y^{2}} \frac{\partial^{2} \Phi}{\partial x \partial y}+\frac{\partial^{3} \Phi}{\partial y^{3}}\left(\frac{\partial^{2} \Phi}{\partial x^{2}}\right)^{2}\right. \\
& \left.-\frac{\partial^{3} \Phi}{\partial x^{2} \partial y}\left(\frac{\partial^{2} \Phi}{\partial x \partial y}\right)^{2}-\frac{\partial^{3} \Phi}{\partial x \partial y^{2}} \frac{\partial^{2} \Phi}{\partial x \partial y} \frac{\partial^{2} \Phi}{\partial x^{2}}\right]\left(z_{c}\right) .
\end{aligned}
$$

Таким образом, $z_{c}$ является критической точкой типа $A_{2}$ при $\partial_{x}^{2} \Phi\left(z_{c}\right) \neq 0$, если выражение, стоящее в квадратных скобках в правой части последнего равенства, отлично от нуля. 


\section{§ 4. Особенности типа $D_{4}^{ \pm}$}

Если хотя бы одна из вторых производных $\partial_{x}^{2} \Phi, \partial_{x} \partial_{y} \Phi, \partial_{y}^{2} \Phi$ больше $C(\lambda) \times$ $\Omega^{-(1-\varepsilon) / 3}$, то оценка ОИ $I_{2}[u](3.1)$ уже получена в $\S 3$. Таким образом, осталось рассмотреть область $T_{2}$ такую, что при $t \in T_{2}$ все вторые производные фазы $\Phi$ малы, точнее, область $T_{2}$, где

$$
\left|\partial^{2} \Phi(z)\right| \leqslant C(\lambda) \Omega^{-(1-\varepsilon) / 3}, \quad t \in T_{2} .
$$

Простейшей после $A_{k}$ и наиболее часто встречающейся является особенность $D_{4}^{ \pm}[20],[21]$. Пусть при $t=0$ точка $z=0$ является критической точкой с особенностью $D_{4}^{ \pm}$. Тогда в этой точке $\partial^{2} \Phi(0)=0$ и $D(0) \neq 0$, где $D(0)-$ значение в точке $z=0$ дискриминанта кубической формы от переменных $x, y$, задаваемой членами третьей степени в ряде Тейлора фазы $\Phi$. В этом случае основной вклад $I_{2}^{0}[u \mid 0]\left(\right.$ см. (1.4)) точки $z=0$ в асимптотику интеграла $I_{2}[u]$ (см. (3.1)) имеет вид

$$
I_{2}^{0}[u \mid 0]=\frac{C[u]}{\Omega^{2 / 3}|D(0)|^{1 / 6}} .
$$

Чтобы записать условие (1.8), введем обозначения

$$
\begin{gathered}
f_{0}(z)=\frac{1}{6} \partial_{x}^{3} \Phi(z), \quad f_{1}(z)=\frac{1}{3} \partial_{x}^{2} \partial_{y} \Phi(z) \\
f_{2}(z)=\frac{1}{3} \partial_{x} \partial_{y}^{2} \Phi(z), \quad f_{3}(z)=\frac{1}{6} \partial_{y}^{3} \Phi(z), \quad z=(x, y) .
\end{gathered}
$$

Дискриминант $D(z)$ кубической формы, задаваемой членами третьей степени в ряде Тейлора фазы $\Phi$ в точке $z$, имеет вид

$$
D(z)=\left(f_{1}^{2} f_{2}^{2}-4 f_{1}^{3} f_{3}+18 f_{0} f_{1} f_{2} f_{3}-4 f_{2}^{3} f_{0}+27 f_{0}^{2} f_{3}^{2}\right)(z) .
$$

В соответствии с гипотезой, изложенной в $\S 1$, в настоящем параграфе будем рассматривать область $T_{2}$ в пространстве параметров $\mathbb{R}^{m}$ такую, что

$$
|D(z)| \geqslant A, \quad t \in T_{2} .
$$

Этого условия достаточно, чтобы при $t \in T_{2}$ все критические точки фазы $\Phi$ имели тип $A_{k}$ или $D_{4}^{ \pm}$.

TеOрема 4.1. Пусть $(u, \Phi) \in \Lambda(v, \lambda)$ при $t \in T_{2}, \mathcal{N} \geqslant 1$ - общее число критических точек и для некоторого $\varepsilon, 0<\varepsilon \leqslant 1$, выполняются условия

$$
\begin{gathered}
\left|\partial^{2} \Phi(z)\right| \leqslant C_{1}(\lambda) \Omega^{-\frac{1}{3}(1-\varepsilon)}, \\
|D(z)| \geqslant A \geqslant C_{2}(\lambda) \Omega^{-\frac{2}{15}(1-\varepsilon)}, \\
\Omega \geqslant C_{3}(\lambda, \varepsilon), \quad g \geqslant C_{4}(\lambda, \varepsilon) \Omega^{-\frac{2}{3}(1-\varepsilon)} A^{-3},
\end{gathered}
$$

где $g$ - величина, определенная в (2.6). Тогда при $t \in T_{2}$

$$
\left|I_{2}[u]\right| \leqslant C(\lambda, \varepsilon) \mathcal{N} v \Omega^{-\frac{2}{3}(1-\varepsilon)} A^{-2}
$$

и все константы $C_{i}(\lambda, \varepsilon), C(\lambda, \varepsilon)$ могут быть явно указаны. 
ДокАЗАтеЛЬство. Рассмотрим произвольную фиксированную точку $t \in T_{2}$ в пространстве параметров $\mathbb{R}^{m}$. Выделим некоторое конечное число $\mathcal{N}_{1} \leqslant \mathcal{N}$ критических точек $z_{m}, m \in\{c\}$. Эти точки и числа $\delta_{m} \geqslant \delta_{0}>0$ всегда могут быть выбраны таким образом, чтобы в области $\left|z-z_{m}\right| \geqslant 2 \delta_{m}$ не было критических точек. Заметим, что в круге $\left|z-z_{m}\right| \leqslant \delta_{m}$ могут содержаться несколько критических точек различных типов. Будем предполагать, что

$$
\left|\partial^{2} \Phi(z)\right| \leqslant a
$$

Константа $a$ так же, как и величины $\delta_{m} \geqslant \delta_{0}>0$, пока не фиксирована.

Рассмотрим гладкое разбиение единицы с центрами в точках $z_{m}$, указанных выше, и масштабами $\delta_{m}$, т. е. введем функции $\rho_{m} \in C_{0}^{\infty}$ такие, что

$$
\begin{gathered}
\rho_{m}(z)= \begin{cases}1, & \text { если }\left|z-z_{m}\right| \leqslant \delta_{m}, \\
0, & \text { если }\left|z-z_{m}\right| \geqslant 2 \delta_{m},\end{cases} \\
\left|\partial^{\alpha} \rho_{m}(z)\right| \leqslant C_{|\alpha|} \delta_{m}^{-|\alpha|},
\end{gathered}
$$

и определим функцию $\widetilde{\rho}$ равенством

$$
1=\sum_{z_{m}} \rho_{m}(z)+\widetilde{\rho}(z)
$$

В силу определения функций $\rho_{m}$ имеем

$$
\widetilde{\rho}(z)= \begin{cases}1, & \text { если }\left|z-z_{m}\right| \geqslant 2 \delta_{m} \text { для любого } z_{m}, \\ 0, & \text { если }\left|z-z_{m}\right| \leqslant \delta_{m} \text { для некоторого } z_{m} .\end{cases}
$$

Запишем ОИ $I_{2}[u]$ (см. (3.1)) в виде

$$
I_{2}[u]=\sum_{z_{m}} I_{2}\left[\rho_{m} u\right]+I_{2}[\widetilde{\rho} u]
$$

и будем отдельно оценивать интегралы $I_{2}[\widetilde{\rho} u]$ и $I_{2}\left[\rho_{m} u\right]$. Так как все выделенные критические точки $z_{m}$ равноправны, то достаточно рассмотреть одну из них; будем предполагать, что для этой точки $z_{m}(t)=0$. По формуле Тейлора

$$
\Phi(z)=\Phi(0)+Q(z)+P(z)+h_{4}(z)
$$

где

$$
\begin{gathered}
Q(z) \equiv Q(x, y)=q_{11} x^{2}+2 q_{12} x y+q_{22} y^{2}, \\
P(z) \equiv P(x, y)=a_{0} x^{3}+a_{1} x^{2} y+a_{2} x y^{2}+a_{3} y^{3}, \\
\left|h_{4}(z)\right|=O\left(|z|^{4}\right) .
\end{gathered}
$$

Здесь и ниже

$$
a_{i}=f_{i}(0)
$$

где $f_{i}$ - функции, определенные в равенстве (4.3). Будем предполагать, что

$$
D(0) \geqslant A>0 .
$$


Это предположение означает, что в точке $z=0$ фаза $\Phi$ может иметь особенность типа $D_{4}^{-}$. Случай $D(0)<-A$ (особенность $D_{4}^{+}$) анализируется точно так же и поэтому рассматриваться не будет.

Наша ближайшая цель состоит в том, чтобы (при определенных условиях для величин $\delta_{m}$ ) доказать оценку

$$
|\nabla \Phi(z)| \geqslant C[\Phi] \delta_{m}^{2} \quad \text { при } \quad|z|=\delta_{m}
$$

и указать константу $C[\Phi]$ в ней.

Сделаем линейную замену координат

$$
\begin{gathered}
z=S \xi, \quad \xi=\left(\xi_{1}, \xi_{2}\right), \quad S=\left(s_{i j}\right), \\
x=s_{11} \xi_{1}+s_{12} \xi_{2}, \quad y=s_{21} \xi_{1}+s_{22} \xi_{2},
\end{gathered}
$$

приводящую кубическую форму $P(x, y)$ (см. (4.12)) к каноническому виду [27], [28]:

$$
P(x, y)=\gamma \xi_{1}\left(\xi_{1}^{2}-\xi_{2}^{2}\right) .
$$

При условии (4.14) полином $P(x, 1)$ имеет три различных действительных корня $\alpha_{1}, \alpha_{2}, \alpha_{3}$, которые будем нумеровать таким образом, что $\alpha_{1} \rightarrow \infty$ при $a_{0} \rightarrow 0$. Тогда из разложения

$$
P(x, y)=a_{0}\left(x-\alpha_{1} y\right)\left(x-\alpha_{2} y\right)\left(x-\alpha_{3} y\right)
$$

следует, что

$$
\begin{array}{ll}
s_{11}=\frac{1}{2}\left(\frac{\alpha_{2}}{\alpha_{2}-\alpha_{3}}-\frac{\alpha_{1}}{\alpha_{3}-\alpha_{1}}\right), & s_{12}=\frac{1}{2}\left(\frac{\alpha_{2}}{\alpha_{2}-\alpha_{3}}+\frac{\alpha_{1}}{\alpha_{3}-\alpha_{1}}\right), \\
s_{21}=\frac{1}{2}\left(\frac{1}{\alpha_{2}-\alpha_{3}}-\frac{1}{\alpha_{3}-\alpha_{1}}\right), & s_{22}=\frac{1}{2}\left(\frac{1}{\alpha_{2}-\alpha_{3}}+\frac{1}{\alpha_{3}-\alpha_{1}}\right) .
\end{array}
$$

При этом

$$
\begin{gathered}
\gamma=\frac{1}{4} \frac{\left(\alpha_{2}-\alpha_{1}\right)^{2}}{\left(\alpha_{2}-\alpha_{3}\right)\left(\alpha_{3}-\alpha_{1}\right)}, \\
D(0)=a_{0}^{4}\left(\alpha_{2}-\alpha_{1}\right)^{2}\left(\alpha_{3}-\alpha_{1}\right)^{2}\left(\alpha_{3}-\alpha_{2}\right)^{2} .
\end{gathered}
$$

Заметим, что если $a_{0} \rightarrow 0$, то

$$
\begin{gathered}
\alpha_{1} \rightarrow-\frac{a_{1}}{a_{0}}, \quad \alpha_{2,3} \rightarrow \frac{-a_{2} \pm \sqrt{a_{2}^{2}-4 a_{1} a_{3}}}{2 a_{1}}, \\
D(0) \rightarrow a_{1}^{2}\left(a_{2}^{2}-4 a_{1} a_{3}\right), \quad \gamma \rightarrow-\frac{a_{1}}{4\left(\alpha_{2}-\alpha_{3}\right)}
\end{gathered}
$$

и все приведенные ниже результаты выдерживают предельный переход $a_{0} \rightarrow 0$. В новых переменных (см. (4.12), (4.15)) имеем

$$
Q(z)=Q(S \xi)=p_{11} \xi_{1}^{2}+2 p_{12} \xi_{1} \xi_{2}+p_{22} \xi_{2}^{2}
$$

Будем предполагать, что

$$
\left|s_{i j}\right| \leqslant s
$$


В силу неравенства (4.8) имеем $\left|q_{i j}\right| \leqslant a$, следовательно,

$$
\begin{gathered}
\left|p_{i j}\right| \leqslant \operatorname{Cas}^{2} \\
\left|\nabla_{\xi} Q(S \xi)\right| \leqslant \operatorname{Cas}^{2}|\xi|, \quad \nabla_{\xi}=\left(\frac{\partial}{\partial \xi_{1}}, \frac{\partial}{\partial \xi_{2}}\right) .
\end{gathered}
$$

Из равенства (4.16) получаем оценку

$$
\left|\nabla_{\xi} P(S \xi)\right| \geqslant C|\gamma||\xi|^{2} .
$$

С другой стороны, используя очевидные неравенства

$$
\begin{gathered}
\left|\nabla_{\xi} x\right| \leqslant C s, \quad\left|\nabla_{\xi} y\right| \leqslant C s, \quad|z| \leqslant 1, \\
\left|\nabla_{z} h_{4}(z)\right| \leqslant C H|z|^{3}, \quad H \leqslant \lambda_{0} \lambda_{1}^{4},
\end{gathered}
$$

имеем

$$
\left|\nabla_{\xi} h_{4}(S \xi)\right| \leqslant C H s|z|^{3} \leqslant C H s^{4}|\xi|^{3} .
$$

Оценки (4.20), (4.21), (4.23) показывают, что при условиях

$$
C \frac{a s^{2}}{|\gamma|} \leqslant|\xi| \leqslant C \frac{|\gamma|}{H s^{4}}
$$

имеет место неравенство

$$
\left|\nabla_{\xi} \Phi(S \xi)\right| \geqslant C|\gamma||\xi|^{2}
$$

Прямым вычислением получаем

$$
\operatorname{det} S=\frac{1}{2} \frac{\left(\alpha_{2}-\alpha_{1}\right)}{\left(\alpha_{2}-\alpha_{3}\right)\left(\alpha_{3}-\alpha_{1}\right)} .
$$

Поскольку $\xi=S^{-1} z$, то

$$
s^{-1}|z| \leqslant|\xi| \leqslant s|\operatorname{det} S|^{-1}|z|,
$$

и из $(4.24),(4.25)$ получаем, что в кольце

$$
C \frac{a s^{3}}{|\gamma|} \leqslant|z| \leqslant C \frac{|\gamma||\operatorname{det} S|}{H s^{5}}
$$

нет критических точек и в этом кольце

$$
|\nabla \Phi(z)| \geqslant C|\gamma| s^{-3}|z|^{2}
$$

Таким образом, если величины $\delta_{m}$ выбраны так, что

$$
\delta_{m} \geqslant C \frac{a s^{3}}{|\gamma|}, \quad \delta_{m} \leqslant C \frac{|\gamma||\operatorname{det} S|}{H s^{5}},
$$

то имеет место нужная нам оценка

$$
|\nabla \Phi(z)| \geqslant C|\gamma| s^{-3} \delta_{m}^{2}, \quad|z|=\delta_{m} .
$$


Заметим, что из приведенных результатов следует ограничение снизу расстояния между выбранными критическими точками $z_{m} \in\left\{z_{c}\right\}$ :

$$
\left|z_{m}-z_{m^{\prime}}\right| \geqslant C \frac{|\gamma||\operatorname{det} S|}{H s^{5}} .
$$

Входящие в условия (4.29) величины $s^{3}|\gamma|^{-1}$ и $|\gamma||\operatorname{det} S| H^{-1} s^{-5}$ можно явно выразить через $|D(0)|$. Для этого, используя выражение (4.18) для $D(0)$, запишем величины $s_{i j}(4.17)$ в виде

$$
s_{i j}=\varphi_{i j}\left(\alpha_{k}\right) a_{0}^{2}\left(\alpha_{2}-\alpha_{1}\right)(D(0))^{-1 / 2} .
$$

Из определения корней $\alpha_{k}$ следует, что

$$
\left|a_{0} \varphi_{i j}\left(\alpha_{k}\right)\right| \leqslant C\left(\lambda_{0} \lambda_{1}^{3}+\left(\lambda_{0} \lambda_{1}^{3}\right)^{1 / 3}\right) .
$$

Таким образом, можно взять

$$
s=C \frac{\left(\lambda_{0} \lambda_{1}^{3}+\left(\lambda_{0} \lambda_{1}^{3}\right)^{1 / 3}\right) a_{0}^{2}\left(\alpha_{2}-\alpha_{1}\right)}{\sqrt{D(0)}} .
$$

Выражения (4.18), (4.26) для величин $|\gamma|$ и $|\operatorname{det} S|$ можно переписать в виде

$$
|\gamma|=\frac{\left(\alpha_{2}-\alpha_{1}\right)^{3}\left(a_{0}\right)^{3}}{\sqrt{D(0)}}, \quad|\operatorname{det} S|=\frac{a_{0}^{2}\left(\alpha_{2}-\alpha_{1}\right)^{2}}{\sqrt{D(0)}},
$$

следовательно,

$$
\frac{s^{3}}{|\gamma|}=\frac{C_{1}(\lambda)}{D(0)}, \quad \frac{|\gamma||\operatorname{det} S|}{s^{5}}=C_{2}(\lambda)(D(0))^{3 / 2} .
$$

Переходим к оценке интеграла $I_{2}[\widetilde{\rho} u]$, входящего в разложение (4.10). Из оценки (4.30) и определения (2.6) величины $g$ получаем, что при условии

$$
g \geqslant C \frac{|\gamma|}{s^{3}} \delta_{m}^{2}
$$

минимум величины $|\nabla \Phi(z)|$ в области $\left|z-z_{m}\right| \geqslant \delta_{m}$ достигается на одной из окружностей $\left|z-z_{m}\right|=\delta_{m}$, и, следовательно, при выполнении условий (4.29), (4.33) оценка (4.30) верна во всей области $\left|z-z_{m}\right| \geqslant \delta_{m}$, т. е.

$$
|\nabla \Phi(z)| \geqslant C|\gamma| s^{-3} \delta_{0}^{2}, \quad\left|z-z_{m}\right| \geqslant \delta_{m} \geqslant \delta_{0}>0 .
$$

В силу равенства (4.9) если $z \in \operatorname{supp} \widetilde{\rho}$, то $\left|z-z_{m}\right| \geqslant \delta_{m}$. Так как в этой области нет критических точек, то, интегрируя $I_{2}[\widetilde{\rho} u]$ по частям (см. формулу (3.14)), имеем

$$
I_{2}[\widetilde{\rho} u]=\left(\frac{i}{\Omega}\right)^{N} \iint\left(A_{\Phi}\right)^{N}(\widetilde{\rho} u) e^{i \Omega \Phi} d x d y .
$$

Далее воспользуемся тривиальной оценкой

$$
\left|I_{2}[\widetilde{\rho} u]\right| \leqslant \frac{1}{\Omega^{N}} \max _{|z| \leqslant 1}\left|\left(A_{\Phi}\right)^{N}(\widetilde{\rho} u)\right|
$$


и для оценки величины $\left(A_{\Phi}\right)^{N}(\widetilde{\rho} u)(z)$ применим лемму 3.1. В интересующем нас случае (в обозначениях этой леммы)

$$
f>\widetilde{\rho} u, \quad \Phi_{1}=C \frac{|\gamma|}{s^{3}} \delta_{0}^{2}, \quad\left|\partial^{2} \Phi\right|=a .
$$

Будем предполагать, что $\delta_{m} \leqslant C \lambda_{2}^{-1}$, и тогда

$$
\left|\partial^{\alpha}(\widetilde{\rho} u)\right| \leqslant C_{|\alpha|} v \mathcal{N}_{1} \delta_{0}^{-|\alpha|}
$$

где $\mathcal{N}_{1}$ - число выбранных критических точек. Если выполняются условия

$$
\delta_{m} \lambda_{1} \leqslant 1, \quad \delta_{m} \lambda_{2} \leqslant 1, \quad \frac{|\gamma|}{s^{3}} \frac{\delta_{m}}{a} \leqslant 1, \quad \frac{\delta_{m}}{a} \lambda_{0} \lambda_{1}^{3} \geqslant 1,
$$

то из оценки (3.16) получим, что для любого $N \geqslant 2$

$$
\left|\left(A_{\Phi}\right)^{N}(\widetilde{\rho} u)(z)\right| \leqslant C_{N} v \mathcal{N}_{1}\left[\frac{s^{3}}{\Omega|\gamma| \delta_{0}^{3}}\left(1+\frac{s^{3} \lambda_{0} \lambda_{1}^{3}}{|\gamma|}\right)\right]^{N},
$$

следовательно (см. (4.35)),

$$
\left|I_{2}[\widetilde{\rho} u]\right| \leqslant C_{N} v \mathcal{N}_{1}\left[\frac{s^{3}}{\Omega|\gamma| \delta_{0}^{3}}\left(1+\frac{s^{3} \lambda_{0} \lambda_{1}^{3}}{|\gamma|}\right)\right]^{N} .
$$

Поскольку из определения дискриминанта $D(0)$ следует, что

$$
|D(0)| \leqslant C\left(\lambda_{0} \lambda_{1}^{3}\right)^{4}
$$

то константу $C$ в (4.31) можно выбрать таким образом, что

$$
\frac{s^{3} \lambda_{0} \lambda_{1}^{3}}{|\gamma|} \geqslant 1
$$

и тогда при условии

$$
\frac{s^{6} \lambda_{0} \lambda_{1}^{3}}{\Omega|\gamma|^{2} \delta_{0}^{3}} \leqslant C \Omega^{-\varepsilon}, \quad \varepsilon>0
$$

имеет место оценка

$$
\left|I_{2}[\widetilde{\rho} u]\right| \leqslant C_{N} v \mathcal{N}_{1} \Omega^{-\varepsilon N} \quad \forall N \geqslant 2 .
$$

Будем считать, что $\delta_{m}=\delta_{0}$, и оставшиеся интегралы $I_{2}\left[\rho_{m} u\right]$ оценим тривиально:

$$
\left|I_{2}\left[\rho_{m} u\right]\right| \leqslant C v \delta_{0}^{2} .
$$

Напомним, что константа $v$ входит в условие (2.2). Теперь прямо из разложения (4.10) получим

$$
\left|I_{2}[u]\right| \leqslant C v \mathcal{N}_{1}\left[\delta_{0}^{2}+C_{N} \Omega^{-\varepsilon N}\right] \quad \forall N \geqslant 2 .
$$

Остается показать, что все условия для величин $\delta_{m}=\delta_{0}$ непротиворечивы, и выбрать нужным образом $\delta_{0}$ и число $N$. С учетом равенств (4.32), условие (4.39) принимает вид

$$
\delta_{0} \geqslant C(\lambda)(D(0))^{-2 / 3} \Omega^{-(1-\varepsilon) / 3} .
$$


Теперь заметим, что в силу исходных предположений константу $a$ в (4.8) можно выбрать так, что

$$
a \leqslant C \lambda_{0}^{2 / 3} \lambda_{3}^{2} \Omega^{-(1-\varepsilon) / 3} \equiv C(\lambda) \Omega^{-(1-\varepsilon) / 3} .
$$

Тогда с учетом равенств (4.32) первое из условий (4.29) и второе из условий (4.36) выполняются, если

$$
\delta_{0} \geqslant C(\lambda)(D(0))^{-1} \Omega^{-(1-\varepsilon) / 3} .
$$

В силу оценки (4.38) условия (4.44), (4.43) выполняются, если выбрать

$$
\delta_{0}=C(\lambda)(D(0))^{-1} \Omega^{-(1-\varepsilon) / 3} .
$$

Рассмотрим оставшиеся условия. Так как константа $H \leqslant \lambda_{0} \lambda_{1}^{4}$ (см. (4.22)), то, снова ссылаясь на равенства (4.32), заключаем, что второе из условий (4.29) выполняется, если выполняется второе из условий (4.6). Условие (4.33) выполняется в силу последнего из условий (4.6). Непосредственно проверяется, что остальные условия, использованные при доказательстве оценки (4.42), в силу выбора $\delta_{0}(4.45)$ и оценки (4.38) имеют вид $\Omega \geqslant C(\lambda)$, следовательно, выполняются в силу третьего из условий (4.6).

С учетом равенства (4.45) оценка (4.42) приобретает вид

$$
\left|I_{2}[u]\right| \leqslant C(\lambda) v \mathcal{N}_{1}\left(A^{-2} \Omega^{-\frac{2}{3}(1-\varepsilon)}+C_{N} \Omega^{-\varepsilon N}\right) .
$$

Поскольку $A \geqslant \Omega^{-\sigma}, \sigma>0$, то, выбирая соответствующим образом $N$, получим нужную оценку (4.7).

Заметим, что оценка (4.46) является неулучшаемой по порядку $\Omega$ при $\varepsilon \rightarrow 0$, но не является асимптотически точной. Для получения асимптотически точной оценки надо еще заменить $A^{-2}$ в (4.6) на $A^{-1 / 6}$. Как уже указывалось во введении, глобальные условия (3.2) на $\partial^{2} \Phi$ и (4.5) на $D(z)$ можно заменить на локальные, т. е. для справедливости полученных результатов достаточно предложить, что условия (3.2), (4.5) выполняются в критических точках $z_{c}$. Таким образом, неисследованной остается область в пространстве параметров, где одновременно для некоторой критической точки $z_{c}$ выполняются условия

$$
\left|\partial^{2} \Phi\left(z_{c}\right)\right| \leqslant C(\lambda) \Omega^{-\frac{1}{3}(1-\varepsilon)}, \quad\left|D\left(z_{c}\right)\right| \leqslant C(\lambda) \Omega^{-\frac{2}{15}(1-\varepsilon)} .
$$

Изложенного выше достаточно, чтобы рассмотреть общие семейства фаз, зависящих не более чем от трех параметров, так как в таких семействах встречаются только особенности типов $A_{k}, D_{4}^{ \pm}$(см. [3], [20], [21]).

В случае четырех параметров могут встретиться еще и особенности $D_{5}^{ \pm}$, а для пяти параметров - особенности типов $D_{6}^{ \pm}, E_{6}^{ \pm}$. Возникает вопрос о возможности описанным выше элементарным методом получить оценки, близкие к асимптотически точным и для этих простых особенностей.

Рассмотрим этот вопрос на примере особенности типа $D_{5}^{ \pm}$. Пусть при $t=0$ точка $z=0$ является критической точкой с особенностью типа $D_{5}^{ \pm}$. Это означает, что $\partial^{2} \Phi(0)=0, D(0)=0$ и полином $P(x, 1)=a_{0} x^{3}+a_{1} x^{2}+a_{2} x+a_{3}$ 
(см. (4.12)) имеет кратные корни. Весь класс особенностей $D_{k}^{ \pm}, k \geqslant 5$, отвечает случаю $\alpha_{1} \neq \alpha_{2}=\alpha_{3}$, а особенность $E_{6}^{ \pm}-$трехкратному корню $\alpha_{1}=\alpha_{2}=\alpha_{3}$. Будем действовать так же, как и выше при анализе особенности $D_{4}^{ \pm}$. Разлагая фазу $\Phi$ в ряд Тейлора в нуле и учитывая члены четвертой степени, имеем

$$
\Phi(z)=\Phi(0)+Q(z)+P(z)+R(z)+h_{5}(z), \quad\left|h_{5}(z)\right|=O\left(|z|^{5}\right),
$$

где

$$
R(z) \equiv R(x, y)=b_{0} x^{4}+b_{1} x^{3} y+b_{2} x^{2} y^{2}+b_{3} x y^{3}+b_{4} y^{4} .
$$

Для простоты предположим, что коэффициент $a_{0}$ в формуле (4.12) для $P(z)$ отличен от нуля $\left(a_{0} \neq 0\right)$. С помощью замены координат

$$
\begin{gathered}
z=S \xi, \quad S=\left(s_{i j}\right), \quad s_{11}=\alpha_{1}\left(\alpha_{1}-\alpha_{2}\right)^{-1}, \\
s_{12}=-\alpha_{2}\left(\alpha_{1}-\alpha_{2}\right)^{-1}, \quad s_{21}=-s_{22}=\left(\alpha_{1}-\alpha_{2}\right)^{-1}
\end{gathered}
$$

приведем полином $P(x, y)$ (см. (4.43)) к каноническому виду $P(S \xi)=a_{0} \xi_{1}^{2} \xi_{2}$. При $D(0)=0$ корни $\alpha_{1} \neq \alpha_{2}=\alpha_{3}$ полинома $P(x, 1)$ явно выписываются как рациональные функции от коэффициентов $a_{i}$.

Введем величины

$$
C_{4}(0)=R\left(s_{12}, s_{22}\right), \quad D_{1}(0)=3 a_{2} a_{0}-a_{1}^{2} .
$$

Аналог формулы (4.2), дающий главный член вклада критической точки $z_{c}=0$ в асимптотику интеграла $I_{2}[u]$, имеет вид

$$
I_{2}^{0}[u \mid 0]=\frac{C[u]}{\Omega^{5 / 8}\left|C_{4}(0)\right|^{1 / 8}\left|D_{1}(0)\right|^{1 / 2}},
$$

и условия $C_{4}(0) \neq 0, D_{1}(0) \neq 0$ необходимы и достаточны для того, чтобы в точке $z=0$ была особенность типа $D_{5}^{ \pm}$. По аналогии с тем, что было сделано выше, надо рассмотреть область параметров $T_{3}$, в которой

$$
C_{4}(z) \neq 0, \quad D_{1}(z)=\left(3 f_{2} f_{0}-f_{1}^{2}\right)(z) \neq 0 .
$$

По определению величина $C_{4}(z)$ получается заменой в выражении $C_{4}(0)$ коэффициентов $a_{i}$ на $f_{i}(4.3)$ и коэффициентов $b_{i}$ на соответствующие четвертые производные $\partial^{4} \Phi(z)$. С помощью нелинейной замены координат $z_{1}=z_{1}(\xi)$, описанной в работе [21], фаза $\Phi$ приводится к виду

$$
\Phi\left(z_{1}\right)=a_{0} y_{1} x_{1}^{2}+C_{4}(0) y_{1}^{4}+h_{5}^{1}\left(z_{1}\right), \quad h_{5}^{1}\left(z_{1}\right)=O\left(\left|z_{1}\right|^{5}\right), \quad z_{1}=\left(x_{1}, y_{1}\right) .
$$

Дальше можно использовать те же рассуждения, что и выше.

В силу теоремы 4.1 нас теперь интересует область параметров $T_{3}$, где $\partial^{2} \Phi=$ $O\left(\Omega^{-\frac{1}{3}(1-\varepsilon)}\right), D(z)=O\left(\Omega^{-\frac{2}{15}(1-\varepsilon)}\right)$. Если использовать грубую оценку $(4.41)$, то это позволяет получить при $t \in T_{3}$ только оценку вида

$$
\left|I_{2}[u]\right| \leqslant C[u, \Phi] \Omega^{-\frac{2}{15}(1-\varepsilon)} .
$$


При $\left|a_{0}\right|>0$ этот результат можно улучшить, более аккуратно оценивая интеграл $I_{2}\left[\rho_{m} u\right]$ с фазой $\Phi\left(z_{1}\right)$. Тогда удается получить оценку

$$
\left|I_{2}[u]\right| \leqslant C[u, \Phi] \Omega^{-\frac{3}{5}(1-\varepsilon)},
$$

которая все еще далека от асимптотически точной (см. (4.48)).

\section{Список литературы}

1. М. В. Федорюк, Метод перевала, Наука, М., 1977.

2. Л. Хермандер, Анализ линейных дифференциальных операторов с частными производными. Т. 1. Теория распределений и анализ Фуръе, Мир, М., 1986; пер. с англ.: L. Hörmander, The analysis of linear partial differential operators. V. I. Distribution theory and Fourier analysis, Grundlehren der Mathematischen Wissenschaften, 256, Springer-Verlag, Berlin, 1983.

3. В.И. Арнольд, "Замечания о методе стационарной фазы и числах Кокстера", УМН, 28:5 (1973), 17-44; англ. пер.: V. I. Arnol'd, "Remarks on the stationary phase method and Coxeter numbers", Russ. Math. Surveys, 28:5 (1973), 19-48.

4. В.И. Арнольд, А.Н. Варченко, С. М. Гусейн-Заде, Особенности дифферениируемых отображсении. Т. 2. Монодромия и асимптотика интегралов, Наука, М., 1984; англ. пер.: V. I. Arnol'd, S. M. Gusein-Zade, A. N. Varchenko, Singularities of differentiable maps. II. Monodromy and the asymptotic behavior of integrals, Birkhäuser, Boston, 1988

5. J. J. Duistermaat, "Oscillatory integrals, Lagrange immersions and unfolding of singularities", Comm. Pure Appl. Math., 27:2 (1974), 207-281.

6. В. Гийемин, С. Стейнберг, Геометрические асимптотики, Мир, М., 1981; пер. с англ.: V. Guillemin, Sh. Stenberg, Geometric asymptotics, Mathematical Surveys, 14, Amer. Math. Soc., Providence, RI, 1977.

7. В.П. Маслов, М.В. Федорюк, Квазиклассическое приближение для уравнений квантовой механики, Наука, М., 1976; англ. пер.: V. P. Maslov and M. V. Fedoryuk, Semi-classical approximation in quantum mechanics, Nauka, Moscow, 1976; English transl. 1981.

8. И. М. Виноградов, Метод тригонометрических сумм в теории чисел, Наука, М., 1971; англ. пер.: I. M. Vinogradov, The method of trigonometric sums in the theory of numbers, Dover Publ., Mineola, NY, 2004.

9. И. М. Виноградов, Особые варианты метода тригонометрических сумм, Наука, M., 1976.

10. J. G. van der Corput, "Neue Zahlentheoretische Abschatzungen", Math. Ann., 89 (1923), 215-254.

11. Г. И. Архипов, А. А. Карацуба, В.Н. Чубариков, "Тригонометрические интегралы", Изв. АН СССР. Сер. матем., 43:5 (1979), 971-1003; англ. пер.: G. I. Arkhipov, A. A. Karatsuba, V. N. Chubarikov, "Trigonometric integrals", Math. USSR Izv., 15:2 (1980), 211-239.

12. Г. И. Архипов, А.А. Карацуба, В.Н. Чубариков, Теория кратных тригонометрических сумм, Наука, М., 1987.

13. G.I Arkhipov, V.N. Chubarikov, A.A. Karatsuba, Trigonometric sums in number theory and analysis, de Gruyter Expositions in Mathematics, 39, Walter de Gruyter, Berlin, 2004.

14. Д. В. Косыгин, А.А. Минасов, Я. Г. Синай, "Стохастические свойства спектров операторов Лапласа-Бельтрами на поверхностях Лиувилля", УМН, 48:4 (1993), 3-130; англ. пер.: D. V. Kosygin, A. A. Minasov, Ya. G. Sinai, "Statistical properties of the spectra of Laplace-Beltrami operators on Liouville surfaces", Russian Math. Surveys, 48:4 (1993), 1-142. 
15. J. Bourgain, " $L^{p}$-estimaties for oscillatory integrals in sevral variables", Geom. Funct. Anal., 1:4 (1991), 321-374.

16. E. M. Stein, Harmonic analysis: real-variable methods, orthogonality, and oscillatory integrals, Princeton Mathematical Series, 43, Princeton Univ. Press, Princeton, NJ, 1993.

17. Д. А. Попов, "Сферическая сходимость ряда и интеграла Фурье индикатора двумерной области", Тр. МИАН, 218 (1997), 354-373; англ. пер.: D. A. Popov, "Spherical convergence of the Fourier series and integral of the indicator of a two-dimensional domain", Proc. Steklov Inst. Math., 218:3 (1997), 352-371.

18. Д. А. Попов, "Оценки с константами для некоторых классов осциллирующих интегралов", УМH, 52:1 (1997), 77-148; англ. пер.: D. A. Popov, "Estimates with constants for some classes of oscillatory integrals", Russian Math. Surveys, 52:1 (1997), $73-145$.

19. Д.А. Попов, "Восстановление характеристических функций в двумерной радоновской томографии", УМH, 53:1 (1998), 115-198; англ. пер.: D. A. Popov, "Reconstruction of characteristic functions in two-dimensional Radon tomography", Russian Math. Surveys, 53:1 (1998), 109-193.

20. В. И. Арнольд, А.Н. Варченко, С. М. Гусейн-Заде, Особенности дифферениируемых отображсении. Т. 1. Классификачия критических точек, каустик и волновых фронтов, Наука, M., 1982; англ. пер.: V. I. Arnol'd, S. M. Gusein-Zade, and A. N. Varchenko, Singularities of differentiable maps. I. Classification of critical points, caustics and wave fronts, Birkhäuser, Boston, 1985.

21. В.И. Арнольд, "Нормальные формы функций вблизи вырожденных критических точек, группы Вейля $A_{k}, D_{k}, E_{k}$ и лагранжевы особенности", Функи. анализ и его прил., 6:4 (1972), 3-25; англ. пер.: V. I. Arnol'd, "Normal forms for functions near degenerate critical points, the Weyl groups of $A_{k}, D_{k}, E_{k}$ and Lagrangian singularities", Funct. Anal. Appl., 6:4 (1972), 254-272.

22. Y. Colin de Verdièr, "Nombre de points entiers dans une famille homothétique de domains de $\mathbb{R}^{n ", ~ A n n . ~ S c i . ~ E ́ c o l e ~ N o r m . ~ S u p . ~(4), ~ 10: 4 ~(1977), ~ 559-575 . ~}$

23. В.Н. Карпушкин, "Равномерные оценки осциллирующих интегралов с гиперболической и параболической фазой”, Тр. семин. им. И.Г. Петровского, 1983, № 9, $3-39$.

24. В. Н. Карпушкин, "Теорема о равномерных оценках осциллирующих интегралов с фазой, зависящей от двух переменных", Тр. сем. им. И.Г. Петровского, 10 (1984), 150-169.

25. В.Н. Карпушкин, "Равномерные оценки объемов", Тр. МИАН, 221, Наука, М., 1998, 225-231; англ. пер.: V. N. Karpushkin, "Uniform estimates of volumes", Proc. Steklov Inst. Math., 221 (1998), 214-220.

26. А. Н. Варченко, "Многогранник Ньютона и оценки осциллирующих интегралов", Функи. анализ и его прил., 10:3 (1976), 1-38; англ. пер.: А. N. Varchenko, "Newton polyhedra and estimation of oscillating integrals", Funct. Anal. Appl., 10:3 (1976), $175-196$.

27. Т. Брёкер, Л. Ландер, Дифферениируемъе ростки и катастрофы, Мир, М., 1977; пер. с англ.: Th. Bröcker, Differentiable germs and catastrophes, London Mathematical Society Lecture Note Series, 17, London, 1975.

28. Г. В. Гуревич, Основы алгебраической теории инвариантов, ГИТТЛ, М., 1948.

Д. А. Попов (D. А. Ророv)

Научно-исследовательский институт

физико-химической биологии им. А. Н. Белозерского,

МГУ им. М. В. Ломоносова
Поступило в редакцию

12.07 .2006

20.09.2007 